\title{
Comparison of PMN-PT and PZN-PT Single-Crystal-Based Ultrasonic Transducers for Nondestructive Evaluation Applications
}

\author{
Yong-Il Kim, Geonwoo Kim, Young-Min Bae ${ }^{1}$, Yeon-Hee Ryu², \\ Kwang-Ja Jeong ${ }^{3}$, Chi-Hwan $\mathrm{Oh}^{4}$ and Ki-Bok Kim* \\ Korea Research Institute of Standards and Science, 267 Gajeong-ro, Daejeon 305-340, Korea \\ ${ }^{1}$ Korea Electrotechnology Research Institute, 111 Hanggaul-ro, Ansan 426-170, Korea \\ ${ }^{2}$ Korea Institute of Oriental Medicine, 1672 Yuseong-daero, Daejeon 305-811, Korea \\ ${ }^{3}$ Daewon Instrument Electric Co., Ltd., 830 Yeonsam-ro, Jeju 690-062, Korea \\ ${ }^{4}$ Daum Energy Co., Ltd., 830 Yeonsam-ro, Jeju 690-062, Korea
}

(Received July 1, 2014; accepted December 9, 2014)

Key words: PZT ceramics, PMN-PT single crystal, PZN-PT single crystal, ultrasonic transducer, nondestructive evaluation

The ultrasonic transducer based on lead zirconate titanate (PZT) ceramics has been widely used for nondestructive evaluation (NDE) applications, but it has crucial limitations in the application of highly attenuative materials. Since lead magnesium niobate-lead titanate (PMN-PT) and lead zinc niobate-lead titanate (PZN-PT) singlecrystal materials offer higher electromechanical coupling coefficients than PZT ceramics, they can present improved bandwidth and sensitivity. Despite their major drawbacks such as a significantly higher cost and difficulties in manufacturing, PMN-PT and PZNPT single crystals will be expected to provide new challenges for NDE applications because of their considerably large piezoelectric coefficients. In this work, we compared the performance characteristics of PMN-PT and PZN-PT single-crystal-based ultrasonic transducers for NDE applications. From the results of Krimholtz, Leedom, and Mattaei (KLM) modeling and simulations, the ultrasonic transducers based on PMN-PT and PZN-PT single crystals with $1 \mathrm{MHz}$ center frequency were fabricated. As a conclusion, there were no significant differences in performance characteristics (signal amplitude and bandwidth) between prototype PMN-PT and PZN-PT single-crystal transducers under the same fabrication conditions.

\section{Introduction}

As a piezoelectric material for ultrasonic transducers for nondestructive evaluation (NDE) applications, the lead zirconate titanate (PZT) ceramic has been widely used owing to its high performance and ease of manufacture. Currently, an ultrasonic

${ }^{*}$ Corresponding author: e-mail: kimkibok@kriss.re.kr 
transducer with a much higher sensitivity is being required for NDE applications, but the PZT ceramic ultrasonic transducer has crucial limitations in the application of highly attenuative materials. In the effort to develop more sensitive ultrasonic transducers for NDE applications, new piezoelectric materials such as lead magnesium niobatelead titanate (PMN-PT) and lead zinc niobate-lead titanate (PZN-PT) single crystals for ultrasonic transducers have been studied. ${ }^{(1-5)}$ Because these relaxor-based single crystals have promising potential for ultrasonic transducer applications, they can offer ultrasonic transducers with significantly improved performance through enhanced bandwidth and sensitivity. ${ }^{(1,6-8)}$ The ultrasonic array transducer based on PMN-PT single-crystal elements for medical imaging was fabricated and compared with ceramic composite arrays. ${ }^{(9)}$ In the field of NDE application, the immersion transducer based on the PMN-PT single crystal was fabricated and compared with PZT-ceramic-based transducers. ${ }^{(4)}$ In our previous work, PMN-PT single-crystal ultrasonic transducers with 2.25 and $5 \mathrm{MHz}$ center frequencies for NDE applications were fabricated and their performance characteristics were compared with those of PZT ceramic and PZTbased 1-3 composite ultrasonic transducers. ${ }^{(10)}$ Despite their major drawbacks such as significantly higher cost and difficulties in manufacture, PMN-PT and PZN-PT single crystals can provide new challenges for NDE applications because of their considerably large piezoelectric coefficients.

In this work, we fabricated two contact-type ultrasonic transducers with PMNPT and PZN-PT single crystals for NDE applications and compared their performance characteristics. Prototype ultrasonic transducers, the center frequency of which was 1 $\mathrm{MHz}$, based on PMN-PT and PZN-PT single crystals were fabricated and their signal amplitudes and bandwidths were compared.

\section{Materials and Methods}

\subsection{Piezoelectric materials}

There are several design parameters for the piezoelectric ultrasonic transducer, such as central frequency, bandwidth, transducer diameter, resolution, near-field length, acoustical focal length, front matching layer, and backing material. ${ }^{(11-14)}$ Among them, the performance of the ultrasonic transducer mainly depends on the type of piezoelectric material. In this study, PMN-28\%PT single crystals were supplied by iBulePhotonics Co., Ltd. (Korea) and PZN-(6-7\%) PT crystals were supplied by Microfine Materials Technologies Pte., Ltd. (Singapore). Table 1 shows the material properties of PMN$\mathrm{PT}^{(10)}$ and PZN-PT single crystals. The material properties of the PZN-PT single crystal except the piezoelectric constant $d_{33}$ were based on the manufacturer's data.

From the table, it is evident that the $d_{33}$ of the PZN-PT single crystal is slightly larger than that of the PMN-PT single crystal. However, it can be observed that the thicknessmode electromechanical coupling coefficient $k_{31}$, which corresponds to the fundamental thickness resonance of a bulk plate poled through its thickness, is higher for the PMNPT single crystal than for the PZN-PT single crystal. The coupling factor $k_{33}$, which corresponds to the length resonance of a thin solid cylinder poled along its axis, is also larger for the PMN-PT single crystal than for the PMN-PT single crystal. From these 
Table 1

Material properties of PMN-28\%PT and PZN-(6-7\%)PT single crystals.

\begin{tabular}{|c|c|c|}
\hline Property & PMN-PT single crystal & PZN-PT single crystal \\
\hline Density $\left(\mathrm{kg} / \mathrm{m}^{3}\right)$ & 8000 & 8350 \\
\hline Dielectric constant after poling at $1 \mathrm{kHz}$ & 5500 & 6500 \\
\hline \multirow{3}{*}{$\begin{array}{lc}\text { Coupling factor } & k_{33} \\
& k_{31} \\
\text { Piezoelectric charge constant } d_{3}(\mathrm{pC} / \mathrm{N})\end{array}$} & 0.92 & 0.90 \\
\hline & 0.67 & 0.50 \\
\hline & 1780 & $2100^{1)}$ \\
\hline Longitudinal wave velocity (m/s) & 3600 & 2750 \\
\hline Acoustic impedance (MRayL) & 28.8 & 22.9 \\
\hline
\end{tabular}

${ }^{1)}$ Value measured using the $d_{33}$ meter.

figures, it may be difficult to distinguish which material is better.

The center frequency of the prototype ultrasonic transducers was about $1 \mathrm{MHz}$. The diameter of the single elements of the PMN-PT and PZN-PT single crystals was 12.7 $\mathrm{mm}$. The calculated thicknesses of the PMN-PT and PZN-PT single-crystal elements for the thickness mode of vibration were 1.80 and $1.38 \mathrm{~mm}$, respectively. The electrical properties of the piezoelectric samples such as impedance and phase were measured using an impedance analyzer (Hewlett Packard, HP4194A, USA) and are shown in Fig. 1. The resonance frequencies of the PMN-PT and PZN-PT single crystals were about 1.10 and $1.35 \mathrm{MHz}$, respectively.

\subsection{Modeling, fabrication, and experiments}

The structure of the piezoelectric ultrasonic transducer is shown in Fig. 2 and its major components are a piezoelectric material, a front matching layer, and a back acoustic layer. ${ }^{(10)}$ The front matching layer or wear plate of the ultrasonic transducer acts as an acoustic transformer between the piezoelectric material and the load material. In this study, considering a highly attenuative material as a load material, the austentitic stainless steel SUS316 was selected. The optimal acoustic impedance of the front matching layer $\left(Z_{2}\right)$ can be determined under the quarter-wavelength propagation condition. ${ }^{(12)}$

$$
Z_{2}=\left(Z_{1} \cdot Z_{3}\right)^{1 / 2}
$$

Here, $Z_{1}$ is the acoustic impedance of the piezoelectric material and $Z_{3}$ is that of the load material.

The acoustic impedance of the load material, SUS316 is about 45 to 50 MRayL. On the basis of eq. (1), the calculated acoustic impedances of the front matching layer were found to be 36.0 to 37.9 MRayL for PMN-PT and 32.1 to 33.8 MRayL for PZN-PT. As a front matching layer, alumina ceramics, the acoustic impedance of which is about 43 MRayL, were selected. The calculated approximate thickness of the front matching layer (alumina disc) for both piezoelectric materials in this study was $2.6 \mathrm{~mm}$. 


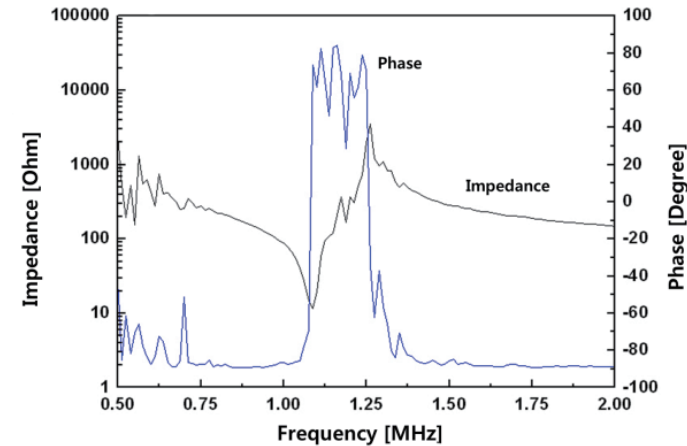

(a)

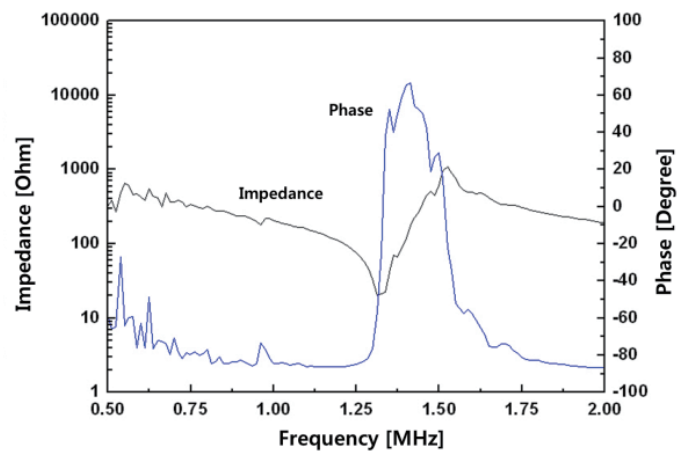

(b)

Fig. 1. (Color online) Measured electrical impedance and phase for (a) PMN-PT single-crystal disc and (b) PZN-PT single-crystal disc.

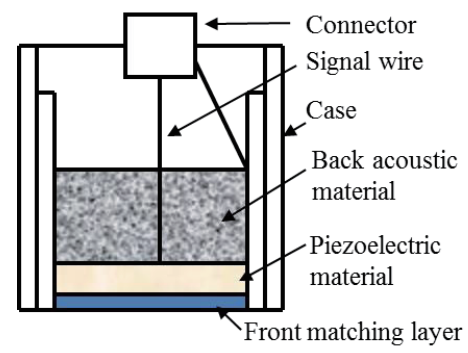

Fig. 2. (Color online) Structure of piezoelectric ultrasonic transducer.

The sensitivity and bandwidth of the piezoelectric ultrasonic transducer depend on the condition of the backing material. In general, the backing material should be highly attenuative and should control the vibration of the transducer by absorbing the energy radiated from the back face of the piezoelectric material. To find the appropriate backing materials for ultrasonic transducers, Krimholtz, Leedom, and Mattaei (KLM) model simulations were performed.(15) Figure 3 shows the description of the transmission line for the piezoelectric transducer in the thickness mode of vibration based on the KLM model. In the KLM model, the electrical and mechanical parts are connected by the electrical transformer. The electrical part consists of the series inductance $L_{\mathrm{S}}$, the electrical transformer ratio $\Phi$, and the capacitances $C_{0}$ and $C^{\prime}$. In Fig. $3, Z_{\mathrm{b}}, Z_{\mathrm{e}}, Z_{\mathrm{p}}, Z_{\text {couple }}$, and $Z_{1}$ are the characteristic acoustic impedances of the back acoustic material, bonding layer, piezoelectric material, coupling material, and load material, respectively.(10)

The simulation conditions of the KLM model for components of both ultrasonic transducers such as epoxy resin, ultrasonic couplant, and load material were similar to those in our previous work. ${ }^{(10)}$ The load material was austenitic SUS316, and the material properties of PMN-PT and PZN-PT single crystals for the KLM model 


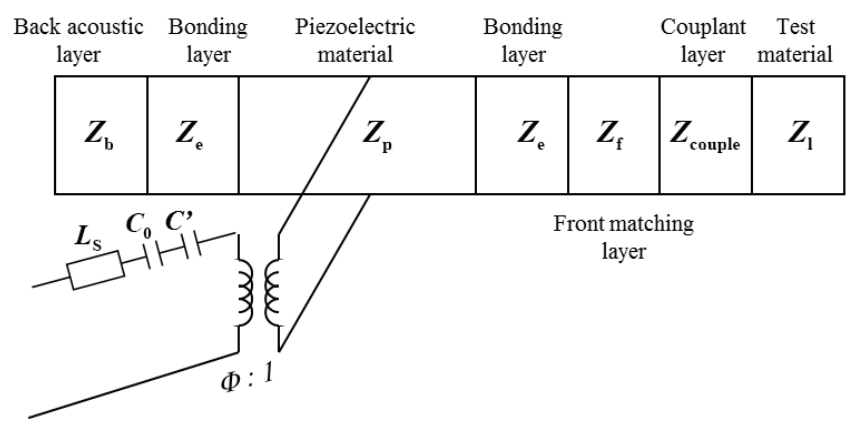

Fig. 3. Description of transmission line for piezoelectric transducer in thickness mode of vibration based on the KLM model. ${ }^{(10)}$

simulation are illustrated in Table 1. In this simulation, the thickness, sound velocity and acoustic impedance of the bonding layer were $1 \mu \mathrm{m}, 2500 \mathrm{~m} / \mathrm{s}$, and $2.7 \mathrm{MRayL}$, respectively. The thickness, sound velocity, and acoustic impedance of the ultrasonic couplant between the load material and the front matching layer were $1 \mu \mathrm{m}, 1650 \mathrm{~m} / \mathrm{s}$, and 1.8 MRayL, respectively. Because the conditions of the front matching layer, bonding layer, and ultrasonic couplant were fixed, the simulation results depended on the effect of the backing materials.

The KLM modeling package (PiezoCAD, Sonic Concepts, Woodinville, WA) was used to analyze the transducer performance under various conditions for the acoustic impedances of the backing material.

Figures 4(a) and 4(b) respectively show the simulation results for pulse-echo waveforms and the frequency spectra of the $1 \mathrm{MHz}$ PMN-PT and PZN-PT transducers at approximately $6 \mathrm{MRayL}$ acoustic impedance of the backing material simulated using PiezoCAD software. The signal shapes, peak amplitudes, and bandwidth of both transducers were very similar. Moreover, it can be considered that no significant differences in signal amplitude between both transducers were found. From the simulation results, as a backing material condition, in this study, the acoustic impedance of the back acoustic material of 6 MRayLwas considered.

The fabrication of the ultrasonic transducer was illustrated in our previous work. ${ }^{(10)}$ From the simulation results for the acoustic impedances of the backing material, epoxy resin and metal powder were mixed. In this study, tungsten powder with a mean particle size of $25 \mu \mathrm{m}$ was selected. The weight ratio of epoxy resin to tungsten powder was 1:2; hence, an acoustic impedance of about 6.5 MRayL was obtained.

Figure 5 shows the finally fabricated $1 \mathrm{MHz}$ prototype ultrasonic transducers. To compare the performance characteristics of both transducers, the back wall echo amplitude and its frequency characteristics were evaluated by the ultrasonic pulse-echo measurement technique. The ultrasonic experimental setup consisting of the prototype ultrasonic transducers, a pulser/receiver (Panametrics, $5601 \mathrm{~A} / \mathrm{T}$ ), an oscilloscope (LeCroy, WaveRunner), a 30-mm-thick test block (Austenite SUS316), and a personal computer was used. ${ }^{(10)}$ 


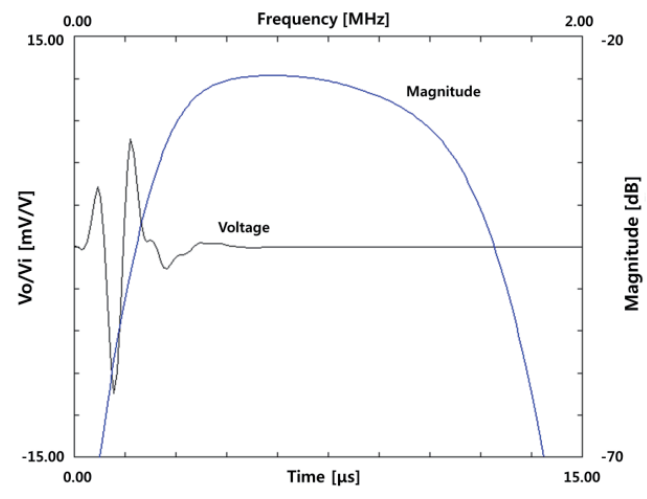

(a)

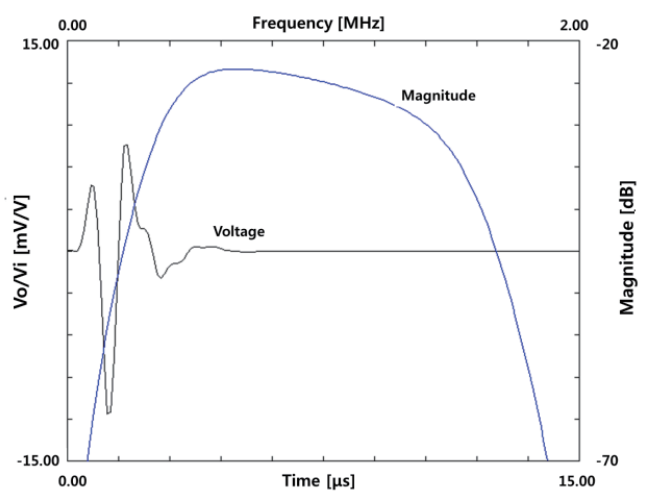

(b)

Fig. 4. (Color online) Comparison of the KLM model simulation results of pulse-echo impulse response for $1 \mathrm{MHz}$ ultrasonic transducers with (a) PMN-PT single crystal and (b) PZN-PT single crystal.

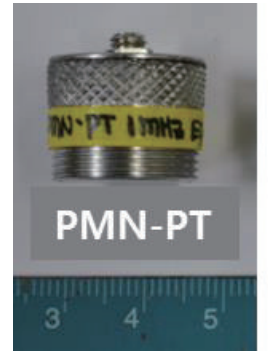

(a)

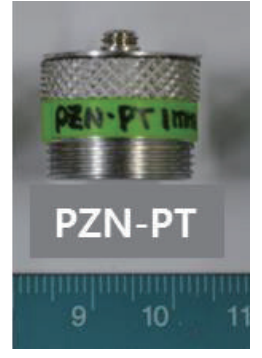

(b)

Fig. 5. (Color online) Photo of fabricated (a) PMN-PT and (b) PZN-PT ultrasonic transducers.

\section{Results and Discussion}

An impulse driving signal from the pulser was applied to the prototype ultrasonic transducer and the reflected signal from the bottom of the test block was received by the transducer. The pulse-echo impulse responses for prototype transducers are shown in Figs. 6 and 7. The performance parameters of the prototype transducers are summarized in Table 2. The peak amplitudes of the first echo signals for the PMN-PT and PZN-PT transducers are about 3.34 and $3.60 \mathrm{~V}$, respectively. The response signal of the PZNPT transducer is slightly greater than that of the PMN-PT transducer. The PZN-PT transducer produces the first echo signal, which is approximately $0.65 \mathrm{~dB}$ better than that of the PMN-PT transducer, but the difference in peak amplitude is only $0.26 \mathrm{~V}$. The center frequencies and bandwidths of the PMN-PT and PZN-PT transducers are 1.26 $\mathrm{MHz}$ and $6.3 \%$ and $1.43 \mathrm{MHz}$ and $8.4 \%$, respectively. From the experimental results, it can be considered that there are no significant differences in signal amplitude or bandwidth between both transducers. 


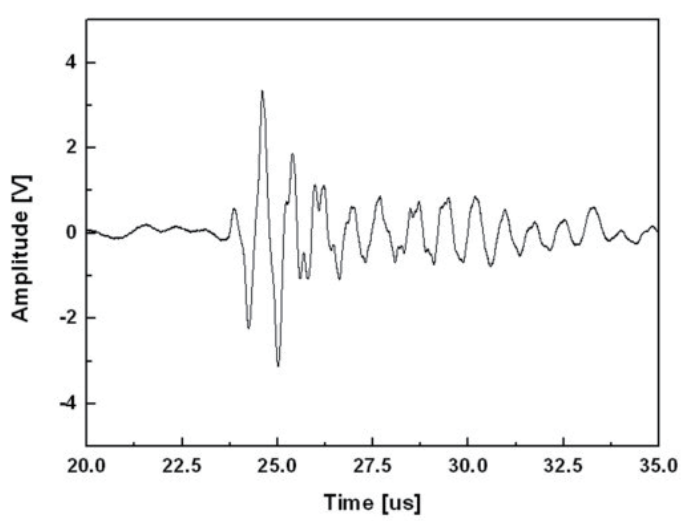

(a)

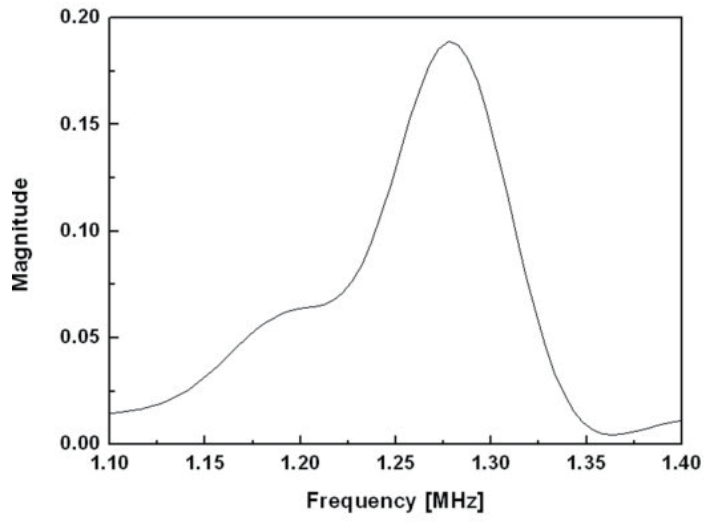

(b)

Fig. 6. (a) Pulse-echo impulse response and (b) frequency spectrum of $1 \mathrm{MHz}$ PMN-PT transducer.

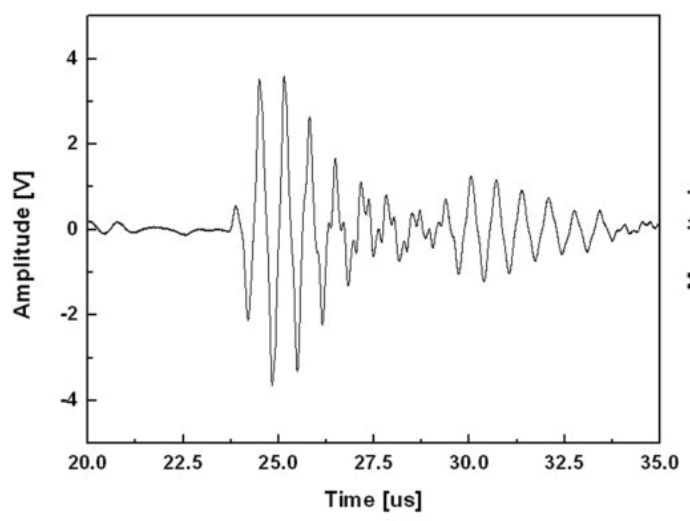

(a)

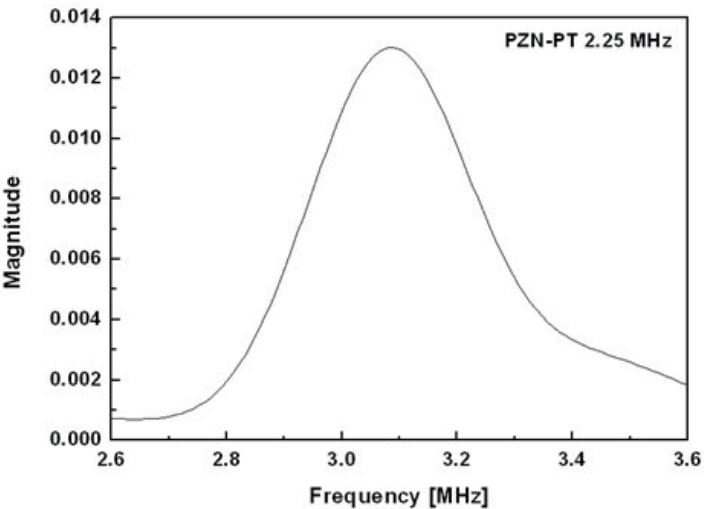

(b)

Fig. 7. (a) Pulse-echo impulse response and (b) frequency spectrum of $1 \mathrm{MHz}$ PZN-PT transducer.

Table 2

Performance parameters of prototype transducers with the $1 \mathrm{MHz}$ PMN-PT and PZN-PT single crystals.

\begin{tabular}{lcc}
\hline \multirow{2}{*}{ Performance parameter } & \multicolumn{2}{c}{ Piezoelectric material } \\
\cline { 2 - 3 } & PMN-PT single crystal & PZN-PT single crystal \\
\hline Peak amplitude (V) & 3.34 & 3.60 \\
Center frequency (MHz) & 1.26 & 1.43 \\
Bandwidth (\%) & 6.3 & 8.4 \\
\hline
\end{tabular}




\section{Conclusions}

In this paper, the simulation, fabrication, and test of the $1 \mathrm{MHz}$ PMN-PT and PZN-PT ultrasonic transducers were described. The optimal conditions of the backing material for each transducer were obtained by KLM model simulation.

The pulse-echo signals for the test material obtained by both transducers showed similar results. Although there is a small difference in piezoelectric properties such as piezoelectric constant and electromechanical coupling factor between PMN-PT and PZNPT single crystals, it is considered that there are no significant differences in performance characteristics such as peak amplitude and bandwidth between both transducers under the same fabrication conditions.

\section{Acknowledgements}

This work was partially supported by the Korea Institute of Energy Technology Evaluation and Planning (KETEP) grant funded by the Ministry of Trade, Industry and Energy (MOTIE) of the Korean government (20131010501810) and the Korea Research Council of Fundamental Science \& Technology (KRCF) grant funded by the Ministry of Science, ICT and Future Planning (MSIP) of the Korean government (P14020).

\section{References}

1 T. Ritter, X. Geng, K. K. Shung, P. D. Lopath, S. S. Park and T. R. Shrout: IEEE Trans. Ultrason. Ferroelectr. Freq. Control 47 (2000) 792.

2 K. C. Cheng, H. L. W. Chan, C. L. Choy, Q. Yin, H. Luo and Z. Yin: IEEE Trans. Ultrason. Ferroelectr. Freq. Control 50 (2003) 1177.

3 P. Marin-Franch, S. Cochran and K. Kirk: J. Mater. Sci. 15 (2004) 715.

4 G. Edwards, H. L. W. Chan, A. Batten, K. H. Lam, H. S. Luo and D. A. Scott: Sen. Actuators, A 132 (2006) 434.

5 Q. Zhou, E. Wu, J. Jin, C. H. Hu, X. Xu, J. Williams, J. M. Cannata, L. Lim and K. K. Shung: IEEE Trans. Ultrason. Ferroelectr. Freq. Control 55 (2008) 1394.

6 S. E. Park and T. R. Shrout: IEEE Trans. Ultrason. Ferroelectr. Freq. Control 12 (1997) 1140.

7 Z. W. Yin and H. S. Luo, P. C. Wang and G. S. Xu: Ferroelectr. 231 (1999) 97.

8 Y. Hosono, Y. Yamashita: J. Electroceram. 17 (2006) 577.

9 D. Robertson, G. Hayward, A. Gachagan and V. Murry: Insight 48 (2006) 97.

10 K. B. Kim, D. K. Hsu, B. Ahn, Y. G. Kim and D. J. Barnard: Ultrasonics 50 (2010) 790.

11 G. Kossoff: IEEE Trans. Son. Ultrason. 13 (1966) 20.

12 C. S. Desilets, J. D. Fraser and G. S. Kino: IEEE Trans. Son. Ultrason. 25 (1978) 115.

13 F. S. Foster and J. W. Hunt: Ultrasonics 16 (1978) 116.

14 J. M. Thijssen, W. A. Verhoef and M. J. Clootermans: Ultrasonics 23 (1985) 41.

15 M. Castillo, P. Acevedo and E. Moreno: Ultrasonics 41 (2003) 671. 